\title{
Unresponsiveness of Experimental Canine Leishmaniosis to a New Amphotericin B Formulation
}

\author{
Leticia Hernández, ${ }^{1}$ Francisco Bolás-Fernández, ${ }^{2}$ Ana Montoya, ${ }^{1}$ Rocío Checa, ${ }^{1}$ \\ Diana Dado, ${ }^{1}$ Rosa Gálvez, ${ }^{1}$ Dolores R. Serrano, ${ }^{3}$ Juan J. Torrado, ${ }^{3}$ Domenico Otranto, ${ }^{4}$ \\ Maria S. Latrofa, ${ }^{4}$ and Guadalupe Miró ${ }^{1}$ \\ ${ }^{1}$ Departamento de Sanidad Animal, Facultad de Veterinaria, Universidad Complutense de Madrid, Avenida Puerta de Hierro s/n, \\ 28040 Madrid, Spain \\ ${ }^{2}$ Departamento de Parasitología, Facultad de Farmacia, Universidad Complutense, Plaza Ramon y Cajal, 28040 Madrid, Spain \\ ${ }^{3}$ Farmacia y Tecnologia Farmaceutica, Facultad de Farmacia, Universidad Complutense de Madrid, Plaza Ramon y Cajal, \\ 28040 Madrid, Spain \\ ${ }^{4}$ Department of Veterinary Medicine, University of Bari, Valenzano, 70010 Bari, Italy
}

Correspondence should be addressed to Guadalupe Miró; gmiro@ucm.es

Received 10 September 2014; Accepted 7 December 2014

Academic Editor: Maria J. Morilla

Copyright (C) 2015 Leticia Hernández et al. This is an open access article distributed under the Creative Commons Attribution License, which permits unrestricted use, distribution, and reproduction in any medium, provided the original work is properly cited.

\begin{abstract}
This study was designed to evaluate the efficacy and safety of a novel free polyaggregated amphotericin B (FPA) formulation used to treat experimental canine leishmaniosis (CanL) caused by Leishmania infantum. Eight healthy beagles were intravenously challenged with $5 \times 10^{7}$ promastigotes per $\mathrm{mL}$ of $L$. infantum. One year after infection, they received an intravenous dose of FPA $(5 \mathrm{mg} / \mathrm{kg})$ every 2 weeks three times. Dogs were assessed monthly for clinical signs, serology, and parasite detection during a followup period of 6 months. Transient adverse effects (i.e., hypotension, diarrhea, bodyweight loss, fever, and asthenia) were observed within 24-48 hours after treatment in 4 animals. In three dogs mean clinical signs scores were reduced. Antibody titers measured by immunofluorescence antibody test (IFAT) had significantly diminished at the end of the study, although according to bone marrow smears and cultures a high percentage of dogs tested positive for the parasite at 6 months posttreatment (PT6). Real-time quantitative PCR (rtQ-PCR) on blood, bone marrow, and urine samples revealed the presence of parasitic DNA in all animals at PT6, although blood loads of the parasite were reduced. These findings indicate that FPA at the dosing regimen used did not achieve clinical or parasitological cure in dogs experimentally infected with L. infantum.
\end{abstract}

\section{Introduction}

Canine leishmaniosis (CanL) is a serious zoonotic disease caused by protozoa of the genus Leishmania (Kinetoplastida, Trypanosomatidae). The disease is endemic in many countries worldwide, including the Mediterranean basin, Portugal, South Africa, and Latin America [1], and is of major veterinary and public health concern since domestic dogs are the main reservoir of Leishmania infantum [2]. Infected female sand flies of the genus Phlebotomus (Diptera, Psychodidae) are the biological vectors for $L$. infantum in Europe. These hematophagous insects transmit the disease by inoculating metacyclic promastigotes in the skin of vertebrate hosts [3].

In dogs, the incubation period of the disease may last from 3 months to several years [4]. Importantly, in areas where the disease is endemic, a large number of infected dogs do not develop clinical signs or clinicopathological abnormalities and are referred to as clinically healthy infected dogs [1]; however, they remain infective to sand flies. CanL is a systemic disease that displays a wide spectrum of clinical signs, such as generalized lymphadenomegaly, weight loss, lethargy, skin lesions, and other less common signs like polyuria/polydypsia, digestive disorders, ocular lesions, and 
lameness or even neurological and cardiorespiratory abnormalities. Common hematological and biochemical findings include nonregenerative anemia, thrombocytopenia, hypergammaglobulinemia, hypoalbuminemia, a reduced albu$\mathrm{min} /$ globulins $(\mathrm{A} / \mathrm{G})$ ratio, and proteinuria/renal azotemia in dogs with renal disease [1,5-7]. CanL is also associated with a high prevalence of chronic renal insufficiency caused by glomerulonephritis, which, in turn, is the main cause of mortality in dogs [8].

Despite recent advances in the diagnosis and control of CanL, the clinical management of sick animals is still a challenge for veterinarians and the antileishmania drugs used over decades are still used today. Although therapy often achieves clinical cure, it does not fully eliminate the parasite or prevent relapse [9], and dogs consequently remain infective to sand flies [10-13].

Today, the combination meglumine antimoniate and/or miltefosine plus allopurinol (a leishmaniostatic agent) is considered the most effective first line treatment for CanL $[9,10,14]$. When combined with allopurinol, both drugs have proved to be more effective $[10,15]$. Miltefosine is the most used in dogs with renal insufficiency [16]. Many other drugs have also been tested including amphotericin $\mathrm{B}(\mathrm{AmB})[17,18]$, aminosidine $[19,20]$, pentamidine [21], metronidazole and spiramycin combined [22], enrofloxacin [23], marbofloxacin [24], ketoconazol [9], and oleyl phosphocholine [25]. More recently, domperidone has been licensed in some European countries as an immunomodulatory drug [26].

$\mathrm{AmB}$ is a polyene macrolide antibiotic derived from the actinomycete Streptomyces nodosus [27]. While traditionally used as an antimycotic, it has also shown activity against protozoal species such as Leishmania and Naegleria [28]. AmB acts by binding primarily to ergosterol in cell membranes, altering their permeability via the formation of aqueous pores permeable to small cations, particularly $\mathrm{K}^{+}$, with the consequence of cell death [29]. AmB is currently the most effective drug for the treatment of human visceral leishmaniosis (VL), and a liposomal AmB formulation is the first line treatment in Europe, United States, and Asia [30, 31]. However, nephrotoxicity caused by renal vasoconstriction and reduced glomerular filtration is the main side effect of $\mathrm{AmB}$ and can lead to renal insufficiency, hypokalemia, and elevated blood urea nitrogen (BUN) and serum creatinine $[32,33]$. In dogs, its use thus requires close monitoring of renal function during treatment. Other common side effects reported have been nausea, vomiting, fever, tremors, and local periphlebitis [34].

With the aim of improving the efficiency of AmB deoxycholate (Fungizona) and reducing its toxicity, new formulations have been developed consisting of carrier systems such as lipid emulsions [17], liposomes [18], nanoparticles [35], and microspheres [36]. However, their high production costs, along with the use of $\mathrm{AmB}$ deoxycholate as the first line treatment for human VL, have limited their use in veterinary medicine.

$\mathrm{AmB}$ desoxycholate diluted in a lipid soya bean oil emulsion for the treatment of dogs naturally infected with CanL $[17,37,38]$ has been reported in several studies to show a high initial clinical efficacy, although anorexia, vomiting, and reversible serum creatinine elevation have been observed $[17,37]$. This formulation also has the drawback of a complex manufacturing process. In another study [18], AmB encapsulated in liposomes (Ambisome) was administered to 13 dogs with natural L. infantum infection. However, although clinical cure was rapid, 12 dogs underwent relapse 4-6 months after treatment and all dogs remained infected.

The aggregation state of a drug and particle size play a role in its toxicity, tissue distribution, and efficacy [39]. During aggregation in liquid medium, AmB molecules self-aggregate to form polyaggregates conferring the final product with a particle size far greater than that of the dimers in conventional AmB. Polyaggregated AmB is much less toxic to mammalian cells $[40,41]$, as observed both in vitro and in vivo (in a murine model) [42-44].

The present study was designed to assess the efficacy and safety of a new cost-effective formulation of $\mathrm{AmB}$, free polyaggregated AmB (FPA), administered intravenously (IV) as three doses $(5 \mathrm{mg} / \mathrm{kg})$ separated by two-week intervals to treat experimentally induced CanL.

\section{Material and Methods}

2.1. Animals. Eight healthy female intact Beagle dogs (8 months old, body weight 10.5 to $16.6 \mathrm{~kg}$ ) were housed in indoor kennels. Each dog was dewormed and vaccinated against rabies, canine distemper, hepatitis/adenovirus type 2, leptospirosis, and parvovirus. All dogs were microchipped and assigned a study number [1-8]. The kennel windows were sprayed with deltamethrin at $25 \mathrm{mg} / \mathrm{m}^{2}$ and fitted with double antimosquito nets to avoid natural Leishmania infection. The dogs were kept in quarantine for 30 days and had daily access to food pellets and water ad libitum. The study protocol was designed according to international guidelines for the Care and Use of Experimental Animals and Spanish Legislation guidelines (RD 1201/2005) and was approved by the Ethics Committee of the Universidad Complutense de Madrid (Madrid, Spain).

The tolerance and pharmacokinetics of the drug formulation under test were assessed in other two female healthy intact Beagle dogs (4 years old, bodyweight 13.1 and $17 \mathrm{~kg}$ ). These animals were separately kept in the same conditions as described above. Before experimental infection and drug administration, it was checked that these animals were negative for L. infantum infection by PCR on blood and bone marrow aspirates and an indirect immunofluorescence antibody test (IFAT, see below). Their good clinical condition was also confirmed in a complete hematological and biochemical evaluation.

2.2. Parasites and Experimental Infection. Details of the experimental infection have been described previously [45]. Briefly, dogs were intravenously infected with $5 \times 10^{7}$ promastigotes of L. infantum (zymodeme MON-1, strain MCRI/ES/06/BCN-721) per $\mathrm{mL}$ in a total volume of $0.5 \mathrm{~mL}$ and were followed for 12 months. 
During one year of follow-up, clinical, immunological, and parasitological examinations were performed monthly. Seroconversion in all animals was produced at 120-180 days postinfection (p.i.). This was confirmed through positive bone marrow cytology and cultures in Novi-McNeal-Nicolle (NNN) media and through the detection of parasite DNA in blood and bone marrow by real-time quantitative PCR (rtQ-PCR). Clinical signs and/or clinicopathological abnormalities compatible with CanL appeared within 120 days after infection. Accordingly, at 180 days p.i., all animals showed clinical signs of CanL.

On Day 360 p.i., the disease in all animals except Dogs 1 and 3 was classified as clinical leishmaniosis stage II according to the system of the Leishvet group [5]. Based on the presence of proteinuria, infection in the latter animals was classified as substage IIb [5].

\subsection{Study Design and Sample Collection}

2.3.1. Infected Dogs. Treatment with FPA was started on Day 390 p.i. Treatment follow-up examinations $(n=9)$ were performed just before the dogs received each of three doses of FPA every two weeks (treatment Days T1, T2, and T3) and monthly thereafter for 6 months (posttreatment Days PT1, PT2, PT3, PT4, PT5, and PT6). Follow-up sessions consisted of a physical exam and clinicopathological, serological, and parasitological tests. Samples were also collected (blood, bone marrow, and urine) to assess the efficacy and safety of treatment (see below).

Peripheral blood samples $(5 \mathrm{~mL})$, obtained by cephalic venipuncture, were distributed into four tubes containing (a) lithium heparin, for biochemical tests, (b) ethylene diamine tetraacetic acid (EDTA) for complete blood counts (CBC), (c) EDTA for rtQ-PCR Leishmania detection, and (d) no additives to determine electrolytes $\left(\mathrm{K}^{+}, \mathrm{Na}^{+}\right.$, and $\left.\mathrm{Cl}^{-}\right)$and for antibody testing (IFAT). The blood variables determined were red blood cell count (RBCC), packed cell volume (PCV), white blood cell count (WBCC), and platelet count. The blood biochemical variables measured to assess renal and hepatic function were urea, creatinine, alanine aminotransferase (ALT), alkaline phosphatase (ALP), total plasma protein, A/G ratio, and serum proteins by electrophoresis.

Urine was collected by catheterization for complete urinalysis including urinary protein creatinine ratio (UPC). An aliquot of urine was used for DNA extraction and quantification.

Bone marrow aspirates $(0.5 \mathrm{~mL})$ were obtained from the fifth-sixth costocondral junction for cytological examination and culture onto NNN medium. An aliquot was stored in buffer NET $10(\mathrm{NaCl} 10 \mathrm{mM}$, EDTA $10 \mathrm{mM}$, and Tris $10 \mathrm{mM})$ at $-20^{\circ} \mathrm{C}$ for DNA extraction.

2.3.2. Healthy Dogs. Blood and urine samples collected from the two female healthy beagles before and after the administration of a single dose of FPA served as controls and were used for the pharmacokinetics study.

\subsection{Treatment}

2.4.1. Formulation. FPA was prepared at the Department of Pharmacy and Pharmaceutical Technology, Faculty of Pharmacy (Universidad Complutense de Madrid), according to the procedure described by Espada et al. [46]. Briefly, $50 \mathrm{mg}$ of AmB (supplied by Azelis, Spain) was dispersed in $5 \mathrm{~mL}$ of an aqueous solution containing $41 \mathrm{mg}$ of sodium deoxycholate (Fluka Chemie A.G., Buchs, Switzerland), $10 \mathrm{mg}$ of dibasic sodium phosphate, and $0.9 \mathrm{mg}$ of monobasic sodium phosphate (Panreac S.A., Barcelona, Spain). Once the drug was homogeneously dispersed, deionised water was added to the resulting mixture up to a final volume of $10 \mathrm{~mL}$, obtaining a final concentration of $5 \mathrm{mg} / \mathrm{mL}$.

2.4.2. Drug Administration. The three $5 \mathrm{mg} / \mathrm{kg}$ doses of FPA given at two-week intervals were administered intravenously by slow perfusion for 10 minutes in an approximate total volume of $40-60 \mathrm{~mL}$, depending on bodyweight, by the investigator until completing a cumulative dose of $12 \mathrm{mg}$. Before receiving FPA, dogs were pretreated with physiological saline solution containing $0.9 \% \mathrm{NaCl}$ (Braun Medical S.A., Madrid, Spain) at a dose of $50 \mathrm{~mL} / \mathrm{kg}$ IV for $15-$ $20 \mathrm{~min}$ to prevent hypokalemia. Prior to administration, FPA $(5 \mathrm{mg} / \mathrm{mL}$ ) was diluted $1: 1: 2$ in $5 \%$ glucose (Braun Medical S.A., Madrid, Spain) and $0.9 \% \mathrm{NaCl}$, respectively, to give a final concentration $1.25 \mathrm{mg} / \mathrm{mL}$ of AmB.

In each dog, consecutive doses were administered via alternating cephalic veins.

The two healthy Beagles used as controls only received a single dose of FPA according to the same procedure.

\subsection{Assessment of Treatment Efficacy}

\subsubsection{Clinical Efficacy}

(a) Physical Examination. At each time point during the treatment period (T1-T3) and 6-month posttreatment period (PT1-PT6), animals were subjected to a complete physical examination. In this exam, body temperature was recorded and animals were weighed and scored for 23 clinical signs using a categorized scoring system from 0 to 3 (from low to high severity), as described in Table 1 (adapted from Miró et al., [15]). By adding the points awarded to each variable, an overall monthly clinical score was calculated (maximum possible score $=52$ ). The clinical response to treatment was assessed by examining changes in clinical score over time as score percentage reductions (PR) calculated using the following equation:

$$
\mathrm{PR}_{i}=100 \times \frac{\left[(\text { score at } \mathrm{T} 1)-\left(\text { score at } \mathrm{PT}_{i}\right)\right]}{(\text { score at } \mathrm{T} 1)},
$$

$i$ is the corresponding day posttreatment.

(b) Clinicopathological Analysis. Each animal was scored for 12 clinicopathological variables (CBC, biochemical profile, electrolytes, urinalysis, and UPC) using a categorized scoring 
TABLE 1: 24 parameters clinical scoring scale of dogs infected with L. infantum.

\begin{tabular}{|c|c|c|c|c|c|}
\hline \multirow{2}{*}{$\begin{array}{l}\text { Condition and } \\
\text { affected organ }\end{array}$} & \multirow{2}{*}{ Clinical sign } & \multicolumn{4}{|c|}{ Severity grade } \\
\hline & & 0 & 1 & 2 & 3 \\
\hline \multirow{4}{*}{ General } & Appetite & Normal & Decreased & Anorexia & - \\
\hline & Asthenia & Absence & Mild & Postration & - \\
\hline & Fatigue & Absence & Mild & Severe & - \\
\hline & Polyuria/Polydipsia & Absence & - & - & Presence \\
\hline \multirow[t]{2}{*}{ Muscles } & Temporal muscles amyotrophy & Absence & Slight temporal amyotrophy & $\begin{array}{l}\text { Marked temporal } \\
\text { amyotrophy }\end{array}$ & - \\
\hline & General amyotrophy & Absence & Slight amyotrphy (emaciation) & Cachexia & - \\
\hline \multirow{2}{*}{ Immune system } & Adenopathy & Absence & Localized & - & Generalized \\
\hline & Splenomegaly & Absence & - & - & Presence \\
\hline \multirow{2}{*}{ Eyes } & Conjunctivitis, blepharitis, and keratitis & Absence & Mild & Severe & - \\
\hline & Uveitis & Absence & - & - & Presence \\
\hline \multirow{3}{*}{ Mucosae } & Pale mucosae & Absence & - & Presence & - \\
\hline & Epistaxis & Absence & - & - & Presence \\
\hline & Oral lesions & Absence & 1 or 2 ulcers and/or nodules & $\begin{array}{l}\text { More than } 2 \text { ulcers } \\
\text { and/or nodules }\end{array}$ & - \\
\hline \multirow{2}{*}{ Digestive system } & Vomiting & Absence & Occasional & Frequent & - \\
\hline & Diarrhea & Absence & Occasional & Frequent or melena & - \\
\hline Joints & Lameness and joint pain & Absence & - & - & Presence \\
\hline \multirow{8}{*}{ Skin } & Erythema & Absence & - & Presence & - \\
\hline & Ulcers & Absence & 1 ulcer or 2 ulcers & $>3$ ulcers & - \\
\hline & Alopecia & Absence & Localized or mild & $\begin{array}{l}\text { Alopecia on more than } \\
25 \% \text { of the body surface }\end{array}$ & - \\
\hline & Exfoliative dermatitis & Absence & Localized & Generalized & - \\
\hline & Hyperkeratosis of nose & Absence & - & Presence & - \\
\hline & Hyperkeratosis of footpads & Absence & - & Presence & - \\
\hline & Generalized hyperkeratosis & Absence & - & Presence & - \\
\hline & Onychogryphosis & Absence & - & Presence & - \\
\hline
\end{tabular}

system from 0 to 2 (maximum possible score of 19) (Table 2). Percentage reductions in scores over time were also calculated.

2.5.2. Serological Follow-Up. Anti-L. infantum immunoglobulin G (IgG) antibodies were detected by IFAT (cut-off 1/100) as described previously [47] on serum dilutions ranging from $1 / 50$ to $1 / 12800$.

\subsubsection{Parasitological Follow-Up}

(1) Microscopy, Molecular Procedures, and Specific Bone Marrow Culture in NNN. The leishmanicidal efficacy of FPA was assessed by microscopy examination of Leishmania amastigotes in three Giemsa-stained bone marrow aspirate smears and promastigotes in bone marrow cultures grown in NNN medium. Blood, bone marrow aspirates, and urine samples collected before and after treatment were subjected to rtQPCR (as described below) at each time point. Stained bone marrow sections were always interpreted by the same operator. Bone marrow aspirates in biphasic NNN cultures [48] were incubated at $27^{\circ} \mathrm{C}$, passaged, and examined weekly under the light microscope over a 4-week period; samples reversing four consecutive negative results were scored as negative [49].

(2) DNA Extraction. DNA from peripheral whole blood samples $(100 \mu \mathrm{L})$, bone marrow aspirates $(100 \mu \mathrm{L})$, and urine $(800 \mu \mathrm{L})$ was extracted using the QuiAamp DNA Micro Kit [50] (QIAGEN), according to the manufacturer's instructions. The extracted DNA was eluted in sterilized water $(70 \mu \mathrm{L})$ and stored at $-20^{\circ} \mathrm{C}$ until use.

(3) Real-Time Quantitative PCR. Over the course of posttreatment follow-up (PT1, PT6), DNA samples were subjected to rtQ-PCR for the simultaneous detection and quantification of $L$. infantum kinetoplast minicircle DNA (kDNA, $120 \mathrm{pb})$ using the primers LEISH-1 (5'-AACTTTTCTGGTCCTCCGGGTAG- $3^{\prime}$ ) and LEISH-2 (5' - ACCCCCAGTTTCCCGCC- $3^{\prime}$ ) and TaqMan-MGB probe (FAM- $5^{\prime}-$ AAAAATGGGTGCAGAAAT- $3^{\prime}$-nonfluorescent quencherMGB) designed by Francino et al. [50]. In a final volume of $20 \mu \mathrm{L}$, the reaction mixture contained $10 \mu \mathrm{L}$ of iQ Supermix 
TABLE 2: Clinicopathological scores recorded in dogs infected with L. infantum.

\begin{tabular}{|c|c|c|c|}
\hline \multirow{2}{*}{ Parameter } & \multicolumn{3}{|c|}{ Severity grade } \\
\hline & 0 & 1 & 2 \\
\hline \multicolumn{4}{|l|}{$\mathrm{CBC}$} \\
\hline Hematocrit/hemoglobin & Normal & Mild anemia & Severe anemia \\
\hline Leukocytes & Normal & Leukocytosis & Leukopenia \\
\hline Platelets & Normal & - & Thrombocytopenia \\
\hline \multicolumn{4}{|l|}{ Biochemical profile } \\
\hline Proteins & Normal & Proteinemia & - \\
\hline Urea & Normal & Elevated & - \\
\hline Creatinine & Normal & Elevated & - \\
\hline Albumin/globulin ratio & Normal & Reduced & - \\
\hline ALT & Normal & Elevated & - \\
\hline \multicolumn{4}{|l|}{ Electrolytes } \\
\hline $\mathrm{K}^{+}, \mathrm{Na}^{+}$, and $\mathrm{Cl}^{-}$ & Normal & - & Increased \\
\hline \multicolumn{4}{|l|}{ Urine } \\
\hline Urine specific gravity & Normal & Reduced & - \\
\hline Proteins & Normal & $100 \mathrm{mg} / \mathrm{dL}$ & $\geq 500 \mathrm{mg} / \mathrm{dL}$ \\
\hline Protein/creatinine ratio & Normal $(<0.5)$ & $>0.5<2$ & $>2$ \\
\hline
\end{tabular}

(Bio-Rad Laboratories, Hercules, CA, USA), each primer at a concentration of $900 \mathrm{nM}$, the probe at a concentration of $200 \mathrm{nM}$, and $2 \mu \mathrm{L}$ of template DNA. The thermal cycling profile consisted of a hot start at $95^{\circ} \mathrm{C}$ for $3 \mathrm{~min}$ and 42 cycles of denaturation $\left(95^{\circ} \mathrm{C}\right.$ for $\left.10 \mathrm{~s}\right)$ and annealing-extension $\left(60^{\circ} \mathrm{C}\right.$ for $30 \mathrm{~s})$. All assays were carried out in duplicate, with negative and positive controls included in each run. rtQPCR was performed in a CFX96 Real-Time System (BioRad Laboratories, Inc., Hercules CA, USA). The fluorescent signal increase was recorded during the extension step of the reaction and the data were analysed using CFX Manager Software Version 1.6 (Bio-Rad).

The amount of Leishmania kDNA in the different samples was determined by the absolute quantification method. A 10fold dilution series of standard DNA from promastigotes (log phase concentration, $1.7 \times 10^{6}$ parasites $/ \mathrm{mL}$ ) of $L$. infantum (zymodeme MON-1) was used to construct a standard curve, and then each dilution was tested in triplicate [51]. The limit of detection of the rtQ-PCR procedure was assessed using a serial dilution from $1.7 \times 10^{-1}$ to $1.7 \times 10^{-8}$ parasites per reaction. Results are expressed as absolute numbers of parasites present in $1 \mathrm{~mL}$ of blood, bone marrow, or urine.

2.6. Safety Assessment. During the course of the study, all adverse events were recorded as the nature of the side effect, time of occurrence, duration, severity, and, if needed, the medical intervention required.

2.7. Statistical Analysis. All data were analyzed using SAS system version 9.2 software. Descriptive statistics were calculated for each set of data (i.e., for each analyte or parameter), including the mean, median, I-III interquartile ranges, standard deviation (SD), minimum and maximum values for quantitative variables, frequencies and percentages for each score class, and other qualitative variables. Since the factors tested before and after treatment were not normally distributed, the nonparametric Wilcoxon signed rank test was used for comparisons between two time points and to explain efficacy criteria. Pretreatment or posttreatment variables were correlated with each other, and degree and significance of correlations were determined through Spearman's rank correlation analysis $(\rho)$. Significance was set at $\alpha=0.05(5 \%)$.

\section{Results}

3.1. Baseline Characteristics. The baseline characteristics of the 8 sick animals before treatment are provided in Table 3 . At the time point $\mathrm{T} 1$, the common clinical signs of CanL observed were generalized lymphadenomegaly $(n=8)$, splenomegaly $(n=7)$, asthenia $(n=3)$, muscular atrophy $(n=3)$, conjunctivitis $(n=6)$, and skin lesions such as erythema $(n=5)$, alopecia $(n=6)$, exfoliative dermatitis $(n=7)$, generalized dermatitis $(n=3)$, hyperkeratosis of the nose $(n=6)$ and of the footpads $(n=8)$, and onychogryphosis $(n=5)$. The mean $( \pm \mathrm{SD})$ clinical score at $\mathrm{T} 1$ was $16.5 \pm 7.6$ out of a maximum of 52 . Dogs 2, 3 , and 7 showed more severe skin lesions increasing their individual scores (to 28, 21, and 25, resp.). Most prominent abnormalities were observed at $\mathrm{T} 1$ including anemia (defined as reduced RBCC, hemoglobinemia, and hematocrit) $(n=7)$ and thrombocytopenia $(n=4)$.

At $\mathrm{T} 1$, all the dogs featured hyperproteinemia (range: 8.5-12 g/dL) and a reduced A/G ratio (range: 0.19-0.55), due to hypoalbuminemia and hypergammaglobulinemia. Other biochemical variables indicating kidney (urea and creatinine) and liver (ALT and ALP) function were normal in most of the dogs. Mild proteinuria was only detected in Dogs 1 and 3 (UPC $=0.97$ and 0.66 , resp.). At this time point, IFAT antibody titers were $1 / 600$ to $1 / 6400$. 


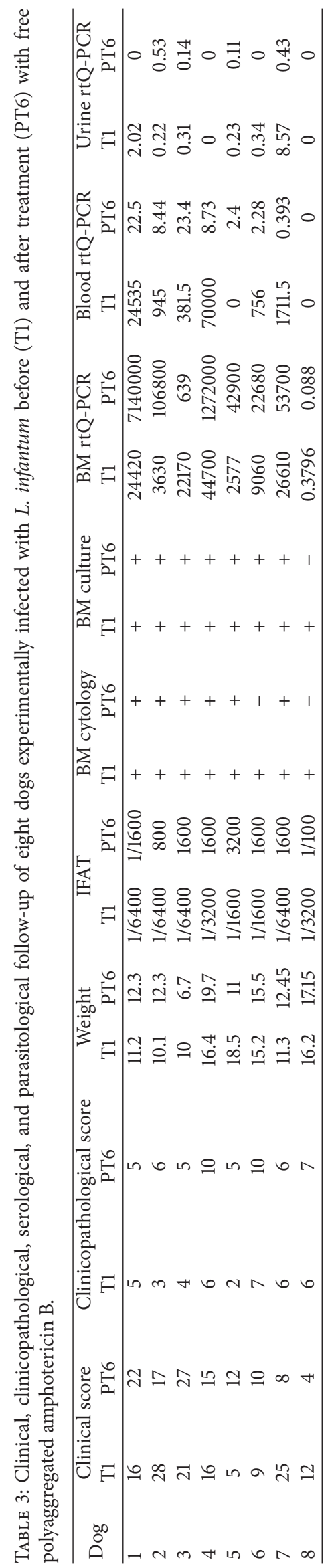


Prior to FPA administration, clinical and laboratory findings in the two healthy Beagles confirmed their good clinical condition.

\subsection{Treatment Efficacy}

3.2.1. Clinical Efficacy. The clinical response to treatment varied in each animal as shown in Table 3. After the first FPA dose, clinical scores in 7 animals increased owing to asthenia, polidypsia, and weight loss. After completing the full course of FPA treatment, the greatest clinical improvement was recorded in Dog 8, lymphadenomegaly and splenomegaly being the only clinical signs at PT6. Dogs 1, 3, and 5 showed no clinical response to treatment and skin lesions, such as alopecia, dermatitis, and generalized hyperkeratosis, even worsened, as did muscular atrophy and lymphadenomegaly.

Over the time period T1 to PT6, the mean percentage reduction in clinical score was $3.55 \pm 60.91 \%$, Dogs 7 and 8 showing the greatest percentage score reductions (65.22 and $61.54 \%$, resp.). In contrast, Dog 5 displayed a clearly worsening clinical picture reflected by a negative percentage score reduction. No significant changes in scores were observed throughout the posttreatment period when compared with scores obtained on Day T1.

Over the 6-month course of follow-up, bodyweights increased though not significantly with the exception of Dogs 3 and 5 that gradually lost weight. Clinical score and bodyweight were negatively correlated at $\operatorname{PT} 1(P=0.016$ and $\rho=-0.8)$ such that animals losing weight showed higher clinical scores.

3.2.2. Clinicopathological Abnormalities. Of the seven animals with anemia on Day T1, three had a hematocrit $<30 \%$. After treatment follow-up, seven dogs remained anemic, and in five of these (Dogs 1, 4, 5, 6 and 7) the hematocrit was $<30 \%$ (mean $=29.03 \% \pm 5.19)$, the reduction between PT3 and PT6 being significant $(P=0.0047)$.

Leukocyte levels remained stable during the study and only Dogs 4, 5, and 6 showed mild leukopenia on day PT6 (the lowest leukocyte count $2800 \times 10^{3} / \mu \mathrm{L}$ in Dog 4). At the end of the study, five animals showed thrombocytopenia (the lowest platelet count $26 \times 10^{3} / \mu \mathrm{L}$ in Dog 4), and only in Dog 8 did this variable return to normal after treatment.

Mean serum concentrations of urea, creatinine, ALT, and ALP did not significantly change during the study, nor were any significant differences recorded in ion levels $\left(\mathrm{K}^{+}, \mathrm{Na}^{+}\right.$, and $\mathrm{Cl}^{-}$). At PT6, the number of dogs still with hyperproteinemia after the monitoring period was only slightly reduced, and only in Dogs 3 and 5 were physiological levels of serum proteins recovered. At the end of the study, all treated dogs still showed severe dysproteinemia: an $A / G$ ratio $<0.3$ was recorded in seven animals. In Dog 8, this ratio increased to 0.61 .

Despite no significant differences detected in the UPC during the study, in response to treatment, five animals $(1,4,5$, 6 , and 7) changed from a nonproteinuric stage to a proteinuric or borderline proteinuric stage, with results ranging from 0.51 (Dog 5 at PT6) to 3.17 (Dog 1 at PT3). In parallel, some dogs

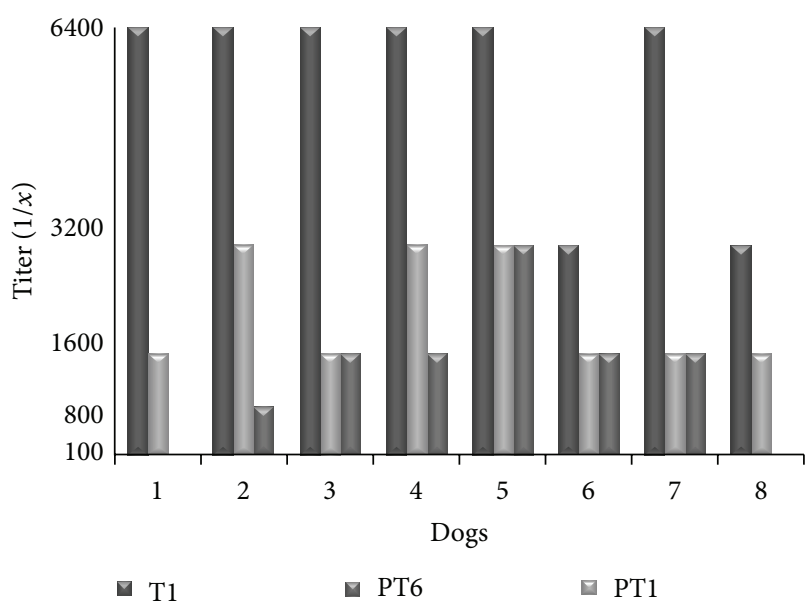

FIGURE 1: Immunofluorescence antibody test (IFAT) titers of antiLeishmania antibodies in eight dogs with experimental leishmaniosis before (T1) and after (PT1 and PT6) treatment with free polyaggregated amphotericin $\mathrm{B}$.

$(3,5,6$, and 8$)$ experienced a slight reduction in urine specific gravity.

No clinicopathological alterations were detected after one day and one week of treatment administration in the two healthy control dogs.

3.2.3. Serological Follow-Up. IFAT anti-L. infantum antibody titers for the 8 animals determined at the nine time points are provided in Figure 1. In all cases, titers were significantly lower at PT6 compared to T1. Six animals showed a steady decrease in antibody levels: in three of these a twofold reduction was observed at PT6, and in Dog 8 a fivefold decrease was detected reaching the cut-off of $1 / 100$. By the end of the study, negative correlation was observed between antibody titer and bodyweight $(P=0.006$ and $\rho=-0.898)$. However, no significant correlation was found between IFAT titer and clinical score.

\subsubsection{Parasitological Follow-Up}

(1) Microscopy and Specific Bone Marrow Culture in NNN. By the end of the study, only Dog 8 scored negative for both bone marrow aspirate cytology and culture (Table 3). Although Leishmania amastigotes were not detected in some animals at some points during the posttreatment period, five out of seven dogs tested at 6 months posttreatment (PT6) returned positive bone marrow aspirate smears and cultures. Positive cytology results correlated positively with culture results both before and after treatment administration $(P<0.0001$ and $\rho=1$ ).

(2) Molecular Procedures. After the six-month follow-up period, all animals remained Leishmania-positive according to some of the samples analyzed by rtQ-PCR. High variation in parasite loads was observed among individuals, both before and after treatment with FPA. This study revealed bone marrow was the tissue varying the most in terms of parasite 


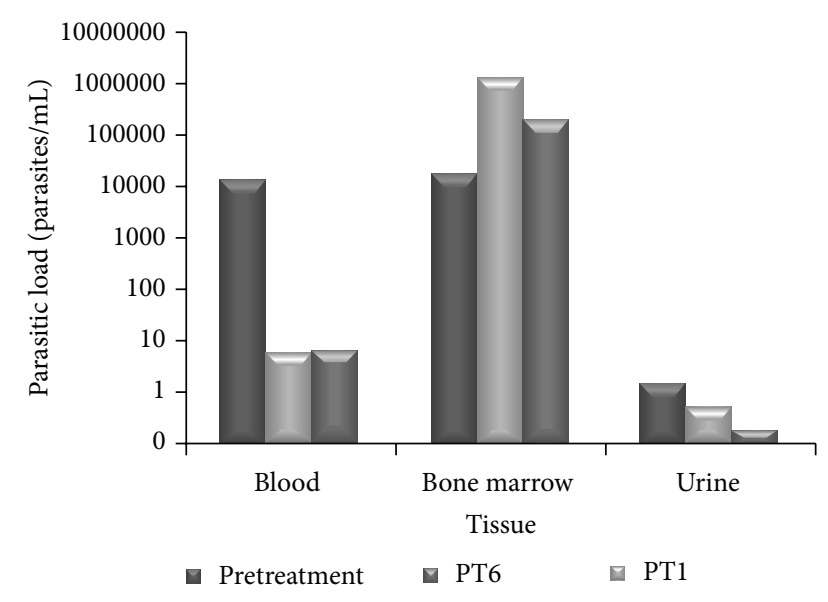

FIgURE 2: Mean parasite loads (parasites $/ \mathrm{mL}$ ) determined by realtime quantitative PCR in blood, bone marrow, and urine samples before treatment (one year p.i.), and one month (PT1) and six months (PT6) after treatment.

numbers among the eight dogs and loads ranged from $8.8 \times$ $10^{-2}$ (Dog 8) to $1.272 \times 10^{6}$ parasites $/ \mathrm{mL}$ (Dog 4) at the end of treatment (PT6). Figure 2 shows the changes produced in mean parasite loads in the different samples tested before and six months after treatment. The bar chart shows a significant drop $(P=0.03)$ in the parasite burden of the blood at the expense of a marked increase in the bone marrow load.

The lowest intensity of infection and the lowest IFAT titers in all samples were recorded in Dog 8. This animal also showed the best clinical response to treatment and was PCRnegative in urine and blood during the entire study period. The remaining animals showed significantly reduced $(P=$ 0.03 ) blood circulating parasite levels except for Dog 5 , which returned a positive PCR result on day PT6 of 2.6 parasites $/ \mathrm{mL}$ of blood.

Contrary to the trend observed in blood, at PT6, bone marrow burdens were still elevated. Despite the presence of rtQ-PCR amplified Leishmania kDNA in the urine of six animals (Dogs 1, 2, 3, 5, 6, and 7) at one year p.i., parasite loads in urine were significantly lower than in the other sample types. Urine parasite loads in Dog 7 before treatment ranged from $1.95 \pm 3.16$ parasites $/ \mathrm{mL}$ to a maximum of 8.57 parasites $/ \mathrm{mL}$. On day PT6 the mean parasite burden fell to $0.3 \pm 0.19$ parasites $/ \mathrm{mL}$; animals 4 and 8 remained PCRnegative and Dog 6 became negative.

3.3. Safety Assessment. At the time of the first dose of FPA given to the experimentally infected Beagles, several events (hypotension, diarrhea, abdominal pain, appetite and bodyweight loss, fever, and asthenia) more likely related to $\mathrm{AmB}$ administration were reported. Hypotension occurred from $15 \mathrm{~min}$ to $1 \mathrm{~h}$ after administration. Dogs 2, 4, 7, and 8 experienced these side effects more frequently after the first dose of FPA. No specific treatment for these episodes was needed and the follow-up of these animals progressed in conformity with the study protocol. Dog 8 did not tolerate the second treatment dose (T2), showing profuse diarrhea, weakness, severe hypotension, and fever $\left(40.1^{\circ} \mathrm{C}\right)$. This animal required complementary treatment with ranitidine (Zantac: $1 \mathrm{mg} / \mathrm{kg} / 12$ hours) and a special diet for gastrointestinal problems (i/d Hills). Because of this reaction, this animal was not given a third dose.

After treatment, the clinical condition of Dog 1 worsened in terms of increased anemia, thrombocytopenia, proteinuria (UPC > 2), and A/G inversion, and at PT5 the animal was prostrated, weak, asthenic, and jaundiced. Blood tests revealed severe nonregenerative anemia, thrombocytopenia, neutrophilia, hypoalbuminemia, and hypergammaglobulinemia. For ethical reasons, this dog was euthanized after PT5. At necropsy, splenomegaly, hepatomegaly, bronchopneumonia, and pericarditis were observed. Histological findings identified the existence of visceral leishmaniosis (with hepatic and splenic lesions, including vacuolar degeneration of hepatocytes, Kuppfer cell hyperplasia with the intracytoplasmic presence of Leishmania amastigotes, hemosiderin accumulation in the splenic capsule, diffuse macrophage hyperplasia with Leishmania amastigote infection, and extramedullary splenic hematopoiesis), and severe fibrinous bronchopneumonia associated with Gram-negative infection. Immunohistochemistry indicated the presence of Leishmania spp. with strong labeling observed in the spleen. The death of this animal was attributed to a nonregenerative anemia, hepatitis, pericarditis, and a serious Gram-negative bacterial lung infection associated with visceral leishmaniosis and pneumonia.

No adverse events were observed in healthy dogs used as controls after the administration of a single FPA dose.

\section{Discussion}

The present clinical trial was designed to evaluate the efficacy and safety of a novel AmB formulation (FPA) for the treatment of experimental CanL.

FPA therapy failed to improve clinical status in the majority of infected dogs. What is more, $50 \%$ of animals showed worsening of clinical signs, especially skin lesions, by the end of the 6-month treatment period. Neither was parasitological cure produced in any of the dogs.

Despite a 100\% success rate for active infection achieved in the eight Beagle dogs, the clinical course of infection and incubation stage varied considerably among the animals. This model reflects the wide clinical response to natural infection [52] described for canine models [53-56]. For this reason, we opted for starting treatment one year after the initial challenge when persistent clinical signs could be detected in every animal.

The increase in clinical scores observed after the first FPA dose administration could be attributed to the fact that a higher dose is needed to reach an effective concentration or due to the side effects recorded in some animals after the first treatment. In response to treatment, clinical improvement was only observed in Dogs 2 and 8 although Dog 8 only received two doses of therapy because of a severe toxic reaction after the second dose. These results differ from those obtained by other authors who used a lipid AmB emulsion 
for the treatment of CanL. With this emulsion of AmB several months after treatment all animals were clinically cured [17, $37,38]$, though two deaths were produced (out of 19) in the study by Lamothe [17]. Comparisons between studies are, however, hindered by the different dosing regimens and the fact that dogs were naturally infected with L. infantum. In addition, the qualitative scoring system used by others is not as accurate as the present system, which includes clinicopathological abnormalities and not only clinical signs.

The main drawbacks of using $\mathrm{AmB}$ to treat CanL are its intravenous administration route, its high cost, and its numerous side effects, particularly nephrotoxicity, since it aggravates the direct harmful effect of CanL on renal function [57]. The clinical efficacy and safety of FPA have been demonstrated in murine models $[39,46,58]$ and in dogs [59]. In a study [39] in which once-weekly doses of $5 \mathrm{mg} / \mathrm{kg}$ FPA were given to mice over a 6-week period, compared with the conventional AmB formulation, high concentrations of $\mathrm{AmB}$ were detected in the liver and spleen, whereas levels in plasma and kidneys were lower. These results suggest that the organs of the mononuclear phagocyte system constitute a major pathway for the accumulation of FPA [39, 43], making infected macrophages more accessible to AmB. In addition, polyaggregates act as an $\mathrm{AmB}$ release system from larger aggregates [59]. The results observed in this murine model prompt further investigation of this formulation in the canine model.

The administration of $5 \mathrm{mg} / \mathrm{kg}$ fortnightly, three times, would offset the fast clearance from the circulation by the mononuclear phagocyte system that occurs with only one dose of FPA, achieving higher effective concentration values.

In trials assessing the treatment of dogs with lipid emulsions of $\mathrm{AmB}$, anorexia, vomiting, and fever were recorded as the most common side effects in $78-81 \%$ of animals, but these gradually resolved $[17,37,60]$. In our study, apart from fever and anorexia, most of the animals suffered (in decreasing order) from hypotension, diarrhea, bodyweight loss, asthenia, and abdominal pain. However, all were self-limiting and also described for AmB treatment in dogs. A single dose of FPA caused no adverse events in healthy Beagle dogs. Regarding the renal toxicity of $\mathrm{AmB}$, some authors describe a transient increase in urea and/or creatinine levels [17, 18, 37, 60]. This was not observed here and levels remained within the normal range. However, five animals became proteinuric (with elevated UPC) after treatment. Proteinuria in these dogs may have been caused by glomerulopathy due to the pathogenic effect of CanL itself, enhancing the nephrotoxic effect of $\mathrm{AmB}$, mainly due to renal vasoconstriction and a reduced glomerular filtration rate [33, 37]. Nevertheless, levels of FPA have been demonstrated to be lower in plasma and kidneys, in comparison with other formulations of AmB [39]. This highlights the need to monitor renal function before and during treatment with $\mathrm{AmB}$, since persistent proteinuria is usually associated with a greater frequency of renal morbidity and mortality [61]. Hence, only CanL patients with minimal or no kidney involvement should receive intravenous FPA treatment.

Dysproteinemia due to hypoalbuminemia and hypergammaglobulinemia is a common finding in CanL [57] and was in fact observed in $100 \%$ of the present dogs at T1. FPA treatment failed to normalize these variables and this has also been observed in clinical trials on allopurinol, allopurinolmeglumine antimoniate [62], or aminosidine [63]. In contrast, miltefosine $[15,64]$ and oleyl phosphocoline [25] were able to rescue these abnormalities.

The humoral response recorded here increased during p.i. follow-up, and antibody titers of 1/6400 were recorded in some animals. This value exceeds that observed in other canine models, though in most of these trials $1 / 640$ was the established limit $[53,65,66]$. Dogs showing higher clinical scores also featured higher antibody levels of the parasite, as confirmed in natural CanL [67-69]. In response to treatment, IFAT-determined titers diminished in six out of eight animals, although a clear correlation with the clinical response was not detected. Some authors have reported clinical improvement in response to treatment with miltefosine, oleyl phosphocholine, or marbofloxacin in the absence of reduced antibody titers determined by IFAT $[16,25,70]$. In contrast, both clinical and serological improvements have been observed for the use of aminosidine [63], enrofloxacin [23], allopurinol [71], miltefosine-allopurinol [15], meglumine antimoniate [72], or meglumine antimoniate-allopurinol [73]. However, most authors consider serology unrepresentative of the clinical progression of treated dogs, at least over a short period $[22,74,75]$, since in a minority of animals antibody titers remain high long after clinical signs have subsided $[13,76]$. Only in Dog 8 did we observe significant correlation between the reduction in antibody levels from $1 / 3200$ to the $1 / 100$ cut-off and a $61.54 \%$ reduction in the clinical score at PT6. This general improvement could be attributed to treatment efficacy but could also reflect a good immune Th2 response and a low intensity of Leishmania infection in this animal, since no DNA was found in blood and urine throughout the study, and bone marrow showed a low parasite burden (less than one parasite/mL at T1, PT1 and PT6). Moreover, as mentioned earlier, this dog did not receive the complete course of treatment and was excluded.

Throughout the year of experimental infection follow-up, a larger number of positive culture results were observed than positive smears, indicating the higher sensitivity of this technique [77]. Overall, the presence of Leishmania was detected in the bone marrow of all animals before treatment. The sensitivity of microscopy examination of bone marrow smears is higher $(60-75 \%)$ than that of lymph nodes $(30-$ $50 \%)[77,78]$, which are enlarged in experimentally infected dogs [79]. rtQ-PCR has a high sensitivity and specificity for the absolute quantification of Leishmania and serves to quantify very low levels of parasites in infected tissues even before seroconversion. This makes this technique useful for diagnosis and treatment monitoring $[10,50,76]$. In our study, the sensitivity of this technique was of 0.001 parasites per PCR reaction. Moreover, its 7-log linear dynamic range allows discrimination from 1 parasite/mL to more than $10^{7}$ parasites/mL of sample [50]. Parasite burdens largely varied according to the animal and tissue (Table 3), bone marrow samples showing the greatest numbers of parasites $/ \mathrm{mL}$, followed by blood, and urine. Indeed, it has been reported that bone marrow contains a larger number of copies of 
Leishmania DNA than peripheral blood [50, 76, 80]. This could be explained by the fact that bone marrow is where invasion and multiplication of the parasite takes place, acting as a target organ, while blood acts as a medium for parasite transport and not as a reservoir organ $[80,81]$. Our rtQ-PCR revealed that FPA did not reduce the parasite load in bone marrow samples and, even one month after treatment, the mean burden in this tissue was still elevated in almost all animals. This could be attributable to a low capacity of FPA to reach the bone marrow or to the high parasite burden in this tissue at the onset of treatment. It should be stressed, however, that the drastic reduction in parasitemia detected at PT1 has significant implications in the fact that animals could be less infective to sand flies at the end of treatment.

Other authors have described the presence of Leishmania kDNA in the urine of dogs contracting natural infection [8284]. In our study, rtQ-PCR detected no significant parasite numbers in urine, suggesting this type of sample is not reliable to quantify Leishmania parasite burden or monitor treatment efficacy. No positive correlation was detected between renal failure and the number of parasites in urine by rtQ-PCR $[82,83]$ probably because we found far fewer parasites in urine than in previous studies [83], the low number of dogs examined, and the fact that none suffered renal azotemia or severe proteinuria.

Although the small number of animals included in this study limits several conclusions, its results indicate that FPA, at the dosing regimen used, was unable to improve the clinical state or reduce hematological and biochemical abnormalities in dogs experimentally infected with L. infantum. A leishmanicidal effect of FPA was produced in blood, but the agent could not lower the parasite load in bone marrow, which could lead to future relapses. The present study shows the null response to $\mathrm{AmB}$ of dogs experimentally infected with L. infantum. Based on these findings, this drug should not be considered for elective treatment of canine leishmaniosis due to its numerous side effects and lack of a clear leishmanicidal effect.

\section{Conflict of Interests}

The authors declare that there is no conflict of interests regarding the publication of this paper.

\section{Acknowledgments}

This work was supported by the Spanish Ministry of Science and Innovation (MICINN) through Grant no. AGL2007$64120 / G A N$. The authors are grateful to Dr. Montserrat Gállego (Laboratory de Parasitologia, Universitat Autònoma de Barcelona) for providing the strain of L. infantum used in the experimental infection. The authors thank Jesús Modesto Moya Ortega for his assistance in the maintenance and welfare of the animals.

\section{References}

[1] G. Baneth, A. F. Koutinas, L. Solano-Gallego, P. Bourdeau, and L. Ferrer, "Canine leishmaniosis-new concepts and insights on an expanding zoonosis: part one," Trends in Parasitology, vol. 24, no. 7, pp. 324-330, 2008.

[2] M. Gramiccia and L. Gradoni, "The current status of zoonotic leishmaniases and approaches to disease control," International Journal for Parasitology, vol. 35, no. 11-12, pp. 1169-1180, 2005.

[3] R. Killick-Kendrick, "Phlebotomine vectors of the leishmaniases: a review," Medical and Veterinary Entomology, vol. 4, no. 1, pp. 1-24, 1990.

[4] R. J. Slappendel, "Canine leishmaniasis. A review based on 95 cases in The Netherlands," Veterinary Quarterly, vol. 10, no. 1, pp. 1-16, 1988.

[5] L. Solano-Gallego, G. Mirá, A. Koutinas et al., "LeishVet guidelines for the practical management of canine leishmaniosis," Parasites and Vectors, vol. 4, article 86, 2011.

[6] A. F. Koutinas, Z. S. Polizopoulou, M. N. Saridomichelakis, D. Argyriadis, A. Fytianou, and K. G. Plevraki, "Clinical considerations on canine visceral leishmaniasis in Greece: a retrospective study of 158 cases (1989-1996)," Journal of the American Animal Hospital Association, vol. 35, no. 5, pp. 376383, 1999.

[7] M. N. Saridomichelakis, "Advances in the pathogenesis of canine leishmaniosis: epidemiologic and diagnostic implications," Veterinary Dermatology, vol. 20, no. 5-6, pp. 471-489, 2009.

[8] F. A. L. Costa, H. Goto, L. C. B. Saldanha et al., "Histopathologic patterns of nephropathy in naturally acquired canine visceral leishmaniasis," Veterinary Pathology, vol. 40, no. 6, pp. 677-684, 2003.

[9] C. Noli and S. T. Auxilia, "Treatment of canine old world visceral leishmaniasis: a systematic review," Veterinary Dermatology, vol. 16, no. 4, pp. 213-232, 2005.

[10] L. Manna, S. Reale, F. Vitale, E. Picillo, L. M. Pavone, and A. E. Gravino, "Real-time PCR assay in Leishmania-infected dogs treated with meglumine antimoniate and allopurinol," Veterinary Journal, vol. 177, no. 2, pp. 279-282, 2008.

[11] F. A. Ikeda-Garcia, R. S. Lopes, F. J. Marques et al., "Clinical and parasitological evaluation of dogs naturally infected by Leishmania (Leishmania) chagasi submitted to treatment with meglumine antimoniate," Veterinary Parasitology, vol. 143, no. 3-4, pp. 254-259, 2007.

[12] R. R. Ribeiro, E. P. Moura, V. M. Pimentel et al., "Reduced tissue parasitic load and infectivity to sand flies in dogs naturally infected by Leishmania (Leishmania) chagasi following treatment with a liposome formulation of meglumine antimoniate," Antimicrobial Agents and Chemotherapy, vol. 52, no. 7, pp. 25642572, 2008.

[13] G. Miró, R. Gálvez, C. Fraile, M. A. Descalzo, and R. Molina, "Infectivity to Phlebotomus perniciosus of dogs naturally parasitized with Leishmania infantum after different treatments," Parasites and Vectors, vol. 4, article 52, 2011.

[14] P. Denerolle and G. Bourdoiseau, "Combination allopurinol and antimony treatment versus antimony alone and allopurinol alone in the treatment of canine leishmaniasis (96 cases)," Journal of Veterinary Internal Medicine, vol. 13, no. 5, pp. 413$415,1999$.

[15] G. Miró, G. Oliva, I. Cruz et al., "Multicentric, controlled clinical study to evaluate effectiveness and safety of miltefosine and allopurinol for canine leishmaniosis," Veterinary Dermatology, vol. 20, no. 5-6, pp. 397-404, 2009.

[16] M. Mateo, L. Maynard, C. Vischer, P. Bianciardi, and G. Miró, "Comparative study on the short term efficacy and adverse 
effects of miltefosine and meglumine antimoniate in dogs with natural leishmaniosis," Parasitology Research, vol. 105, no. 1, pp. 155-162, 2009.

[17] J. Lamothe, "Activity of amphotericin B in lipid emulsion in the initial treatment of canine leishmaniasis," Journal of Small Animal Practice, vol. 42, no. 4, pp. 170-175, 2001.

[18] G. Oliva, L. Gradoni, P. Ciaramella et al., "Activity of liposomal amphotericin B (AmBisome) in dogs naturally infected with Leishmania infantum," Journal of Antimicrobial Chemotherapy, vol. 36, no. 6, pp. 1013-1019, 1995.

[19] G. Oliva, L. Gradoni, L. Cortese et al., "Comparative efficacy of meglumine antimoniate and aminosidine sulphate, alone or in combination, in canine leishmaniasis," Annals of Tropical Medicine and Parasitology, vol. 92, no. 2, pp. 165-171, 1998.

[20] J. A. Vexenat, P. L. Olliaro, J. A. F. de Castro et al., "Clinical recovery and limited cure in canine visceral leishmaniasis treated with aminosidine (paromomycin)," The American Journal of Tropical Medicine and Hygiene, vol. 58, no. 4, pp. 448-453, 1998.

[21] A. Rhalem, H. Sahibi, N. Guessous-Idrissi et al., "Immune response against Leishmania antigens in dogs naturally and experimentally infected with Leishmania infantum," Veterinary Parasitology, vol. 81, no. 3, pp. 173-184, 1999.

[22] M. G. Pennisi, M. de Majo, M. Masucci, D. Britti, F. Vitale, and R. Del Maso, "Efficacy of the treatment of dogs with leishmaniosis with a combination of metronidazole and spiramycin," Veterinary Record, vol. 156, no. 11, pp. 346-349, 2005.

[23] P. Bianciardi, A. Fasanella, V. F. Manzillo et al., "The efficacy of enrofloxacin, alone or combined with metronidazole, in the therapy of canine leishmaniasis," Parasitology Research, vol. 93, no. 6, pp. 486-492, 2004.

[24] S. Rougier, I. Vouldoukis, S. Fournel, S. Pérès, and F. Woehrlé, "Efficacy of different treatment regimens of marbofloxacin in canine visceral leishmaniosis: a pilot study," Veterinary Parasitology, vol. 153, no. 3-4, pp. 244-254, 2008.

[25] L. Hernández, R. Gálvez, A. Montoya et al., "First study on efficacy and tolerability of a new alkylphosphocholine molecule (oleylphosphocholine-OlPC) in the treatment of canine leishmaniosis due to Leishmania infantum," Parasitology Research, vol. 113, no. 1, pp. 157-164, 2014.

[26] P. Gómez-Ochoa, J. A. Castillo, M. Gascón, J. J. Zarate, F. Alvarez, and C. G. Couto, "Use of domperidone in the treatment of canine visceral Leishmaniasis: a clinical trial," Veterinary Journal, vol. 179, no. 2, pp. 259-263, 2009.

[27] J. D. Dutcher, M. B. Young, J. H. Sherman, W. Hibbits, and D. R. Walters, "Chemical studies on amphotericin B. I. Preparation of the hydrogenation product and isolation of mycosamine, an acetolysis product," Antibiotics Annual, pp. 866-869, 1956.

[28] G. Baneth and S. E. Shaw, "Chemotherapy of canine leishmaniosis," Veterinary Parasitology, vol. 106, no. 4, pp. 315-324, 2002.

[29] S. C. Kinsky, "Antibiotic interaction with model membranes," Annual Review of Pharmacology, vol. 10, pp. 119-142, 1970.

[30] F. Chappuis, S. Sundar, A. Hailu et al., "Visceral leishmaniasis: what are the needs for diagnosis, treatment and control?" Nature Reviews Microbiology, vol. 5, no. 11, pp. 873-882, 2007.

[31] S. Sundar, P. K. Sinha, M. Rai et al., "Comparison of short-course multidrug treatment with standard therapy for visceral leishmaniasis in India: An open-label, non-inferiority, randomised controlled trial," The Lancet, vol. 377, no. 9764, pp. 477-486, 2011.

[32] J. D. Berman, G. Ksionski, W. L. Chapman, V. B. Waits, and W. L. Hanson, "Activity of amphotericin B cholesterol dispersion (Amphocil) in experimental visceral leishmaniasis," Antimicrobial Agents and Chemotherapy, vol. 36, no. 9, pp. 19781980, 1992.

[33] R. Laniado-Laborín and M. N. Cabrales-Vargas, "Amphotericin B: side effects and toxicity," Revista Iberoamericana de Micologia, vol. 26, no. 4, pp. 223-227, 2009.

[34] P. Ciaramella and M. Corona, "Canine leishmaniasis: therapeutic aspects," Compendium of Continuing Education for the Practicing Veterinarian, vol. 25, pp. 370-375, 2003.

[35] P. M. Daftarian, G. W. Stone, L. Kovalski et al., "A targeted and adjuvanted nanocarrier lowers the effective dose of liposomal amphotericin B and enhances adaptive immunity in murine cutaneous leishmaniasis," Journal of Infectious Diseases, vol. 208, no. 11, pp. 1914-1922, 2013.

[36] J. A. Sánchez-Brunete, M. A. Dea, S. Rama et al., "Treatment of experimental visceral leishmaniasis with amphotericin B in stable albumin microspheres," Antimicrobial Agents and Chemotherapy, vol. 48, no. 9, pp. 3246-3252, 2004.

[37] O. Cortadellas, "Initial and long-term efficacy of a lipid emulsion of amphotericin B desoxycholate in the management of canine leishmaniasis," Journal of Veterinary Internal Medicine, vol. 17, no. 6, pp. 808-812, 2003.

[38] J. Moreno, J. Nieto, C. Chamizo et al., "The immune response and PBMC subsets in canine visceral leishmaniasis before, and after, chemotherapy," Veterinary Immunology and Immunopathology, vol. 71, no. 3-4, pp. 181-195, 1999.

[39] R. Espada, S. Valdespina, G. Molero, M. A. Dea, M. P. Ballesteros, and J. J. Torrado, "Efficacy of alternative dosing regimens of poly-aggregated amphotericin B," International Journal of Antimicrobial Agents, vol. 32, no. 1, pp. 55-61, 2008.

[40] K. Bartlett, E. Yau, S. C. Hartsel et al., "Effect of heat-treated amphotericin B on renal and fungal cytotoxicity," Antimicrobial Agents and Chemotherapy, vol. 48, no. 1, pp. 333-336, 2004.

[41] E. W. M. van Etten, W. van Vianen, P. Roovers, and P. Frederik, "Mild heating of amphotericin B-desoxycholate: effects on ultrastructure, in vitro activity and toxicity, and therapeutic efficacy in severe candidiasis in leukopenic mice," Antimicrobial Agents and Chemotherapy, vol. 44, no. 6, pp. 1598-1603, 2000.

[42] A. H. A. Mohamed-Ahmed, K. A. Les, K. Seifert, S. L. Croft, and S. Brocchini, "Noncovalent complexation of amphotericinB with poly $(\alpha$-glutamic acid)," Molecular Pharmaceutics, vol.10, no. 3, pp. 940-950, 2013.

[43] R. Espada, S. Valdespina, M. A. Dea et al., "In vivo distribution and therapeutic efficacy of a novel amphotericin B polyaggregated formulation," Journal of Antimicrobial Chemother$a p y$, vol. 61, no. 5, pp. 1125-1131, 2008.

[44] F. Gaboriau, M. Chéron, C. Petit, and J. Bolard, "Heat-induced superaggregation of amphotericin $\mathrm{B}$ reduces its in vitro toxicity: a new way to improve its therapeutic index," Antimicrobial Agents and Chemotherapy, vol. 41, no. 11, pp. 2345-2351, 1997.

[45] L. Hernández, A. Montoya, R. Checa et al., "Course of experimental infection of canine leishmaniosis: follow-up and utility of noninvasive diagnostic techniques," Veterinary Parasitology, vol. 207, no. 1-2, pp. 149-155, 2015.

[46] R. Espada, S. Valdespina, C. Alfonso, G. Rivas, M. P. Ballesteros, and J. J. Torrado, "Effect of aggregation state on the toxicity of different amphotericin B preparations," International Journal of Pharmaceutics, vol. 361, no. 1-2, pp. 64-69, 2008. 
[47] F. Mancianti and N. Meciani, "Specific serodiagnosis of canine leishmaniasis by indirect immunofluorescence, indirect hemagglutination, and counterimmunoelectrophoresis," The American Journal of Veterinary Research, vol. 49, no. 8, pp. 1409-1411, 1988.

[48] WHO, "Report of a meeting of the WHO Expert Committee on the control of leishmaniases," WHO Technical Report Series, WHO, Geneva, Switzerland, 2010.

[49] J. P. Dedet, F. Pratlong, R. Pradinaud, and B. Moreau, "Delayed culture of Leishmania in skin biopsies," Transactions of the Royal Society of Tropical Medicine and Hygiene, vol. 93, no. 6, pp. 673674, 1999.

[50] O. Francino, L. Altet, E. Sánchez-Robert et al., "Advantages of real-time PCR assay for diagnosis and monitoring of canine leishmaniosis," Veterinary Parasitology, vol. 137, no. 3-4, pp. 214221, 2006.

[51] F. Dantas-Torres, M. S. Latrofa, and D. Otranto, "Quantification of Leishmania infantum DNA in females, eggs and larvae of Rhipicephalus sanguineus," Parasites and Vectors, vol. 4, no. 1, article 56, 2011.

[52] M. Hommel, C. L. Jaffe, B. Travi, and G. Milon, "Experimental models for leishmaniasis and for testing anti-leishmanial vaccines," Annals of Tropical Medicine and Parasitology, vol. 89, supplement 1, pp. 55-73, 1995.

[53] J. Fernández-Cotrina, V. Iniesta, S. Belinchón-Lorenzo et al., "Experimental model for reproduction of canine visceral leishmaniosis by Leishmania infantum," Veterinary Parasitology, vol. 192, no. 1-3, pp. 118-128, 2013.

[54] J. Poot, M. E. Rogers, P. A. Bates, and A. Vermeulen, "Detailed analysis of an experimental challenge model for Leishmania infantum (JPC strain) in dogs," Veterinary Parasitology, vol. 130, no. 1-2, pp. 41-53, 2005.

[55] B. L. Travi, E. Y. Osorio, O. A. Saldarriaga et al., "Clinical, parasitologic, and immunologic evolution in dogs experimentally infected with sand fly-derived Leishmania chagasi promastigotes," The American Journal of Tropical Medicine and Hygiene, vol. 81, no. 6, pp. 994-1003, 2009.

[56] A. Rodríguez-Cortés, A. Ojeda, L. López-Fuertes et al., "A long term experimental study of canine visceral leishmaniasis," International Journal for Parasitology, vol. 37, no. 6, pp. 683-693, 2007.

[57] L. Solano-Gallego, A. Koutinas, G. Miró et al., "Directions for the diagnosis, clinical staging, treatment and prevention of canine leishmaniosis," Veterinary Parasitology, vol. 165, no. 1-2, pp. 1-18, 2009.

[58] J. A. Sánchez-Brunete, M. A. Dea, S. Rama et al., "Amphotericin B molecular organization as an essential factor to improve activity/toxicity ratio in the treatment of visceral leishmaniasis," Journal of Drug Targeting, vol. 12, no. 7, pp. 453-460, 2004.

[59] D. R. Serrano, L. Hernández, L. Fleire et al., "Hemolytic and pharmacokinetic studies of liposomal and particulate amphotericin B formulations," International Journal of Pharmaceutics, vol. 447, no. 1-2, pp. 38-46, 2013.

[60] I. Bekersky, G. W. Boswell, R. Hiles, R. M. Fielding, D. Buell, and T. J. Walsh, "Safety and toxicokinetics of intravenous liposomal amphotericin B (AmBisome) in beagle dogs," Pharmaceutical Research, vol. 16, no. 11, pp. 1694-1701, 1999.

[61] M. Pierantozzi, X. Roura, S. Paltrinieri, M. Poggi, and A. Zatelli, "Variation of proteinuria in dogs with leishmaniasis treated with meglumine antimoniate and allopurinol: a retrospective study," Journal of the American Animal Hospital Association, vol. 49, no. 4, pp. 231-236, 2013.
[62] S. Martínez-Subiela, L. J. Bernal, and J. J. Cerón, “Serum concentrations of acute-phase proteins in dogs with leishmaniosis during short-term treatment," The American Journal of Veterinary Research, vol. 64, no. 8, pp. 1021-1026, 2003.

[63] L. V. Athanasiou, M. N. Saridomichelakis, V. I. Kontos, G. Spanakos, and T. S. Rallis, "Treatment of canine leishmaniosis with aminosidine at an optimized dosage regimen: a pilot open clinical trial," Veterinary Parasitology, vol. 192, no. 1-3, pp. 91-97, 2013.

[64] V. Woerly, L. Maynard, A. Sanquer, and H.-M. Eun, “Clinical efficacy and tolerance of miltefosine in the treatment of canine leishmaniosis," Parasitology Research, vol. 105, no. 2, pp. 463469, 2009.

[65] J. Carcelén, V. Iniesta, J. Fernández-Cotrina et al., “The chimerical multi-component Q protein from Leishmania in the absence of adjuvant protects dogs against an experimental Leishmania infantum infection," Vaccine, vol. 27, no. 43, pp. 5964-5973, 2009.

[66] A. C. Rosypal, G. C. Troy, R. B. Duncan, A. M. Zajac, and D. S. Lindsay, "Utility of diagnostic tests used in diagnosis of infection in dogs experimentally inoculated with a North American isolate of Leishmania infantum infantum," Journal of Veterinary Internal Medicine, vol. 19, no. 6, pp. 802-809, 2005.

[67] P. Abranches, G. Santos-Gomes, N. Rachamim, L. Campino, L. F. Schnur, and C. L. Jaffe, "An experimental model for canine visceral leishmaniasis," Parasite Immunology, vol. 13, no. 5, pp. 537-550, 1991.

[68] E. Pinelli, R. Killick-Kendrick, J. Wagenaar, W. Bernadina, G. Del Real, and J. Ruitenberg, "Cellular and humoral immune responses in dogs experimentally and naturally infected with Leishmania infantum," Infection and Immunity, vol. 62, no. 1, pp. 229-235, 1994.

[69] A. B. Reis, O. A. Martins-Filho, A. Teixeira-Carvalho et al., "Parasite density and impaired biochemical/hematological status are associated with severe clinical aspects of canine visceral leishmaniasis," Research in Veterinary Science, vol. 81, no. 1, pp. 68-75, 2006.

[70] S. Rougier, L. Hasseine, P. Delaunay, G. Michel, and P. Marty, "One-year clinical and parasitological follow-up of dogs treated with marbofloxacin for canine leishmaniosis," Veterinary Parasitology, vol. 186, no. 3-4, pp. 245-253, 2012.

[71] A. F. Koutinas, M. N. Saridomichelakis, M. E. Mylonakis et al., "A randomised, blinded, placebo-controlled clinical trial with allopurinol in canine leishmaniosis," Veterinary Parasitology, vol. 98, no. 4, pp. 247-261, 2001.

[72] I. Amusategui, A. Sainz, and M. A. Tesouro, "Effects of antimonial therapy for canine leishmaniasis on antibody titer," Annals of the New York Academy of Sciences, vol. 849, pp. 444-446, 1998.

[73] M. Torres, M. Bardagí, X. Roura, G. Zanna, I. Ravera, and L. Ferrer, "Long term follow-up of dogs diagnosed with leishmaniosis (clinical stage II) and treated with meglumine antimoniate and allopurinol," Veterinary Journal, vol. 188, no. 3, pp. 346-351, 2011.

[74] L. Ferrer, M. J. Aisa, X. Roura, and M. Portús, "Serological diagnosis and treatment of canine leishmaniasis," Veterinary Record, vol. 136, no. 20, pp. 514-516, 1995.

[75] L. Solano-Gallego, P. Morell, M. Arboix, J. Alberola, and L. Ferrer, "Prevalence of Leishmania infantum infection in dogs living in an area of canine Leishmaniasis endemicity using PCR on several tissues and serology," Journal of Clinical Microbiology, vol. 39, no. 2, pp. 560-563, 2001. 
[76] V. Martínez, J. Quilez, A. Sanchez, X. Roura, O. Francino, and L. Altet, "Canine leishmaniasis: the key points for qPCR result interpretation," Parasites and Vectors, vol. 4, article 57, 2011.

[77] J. Alvar, C. Cañavate, R. Molina, J. Moreno, and J. Nieto, "Canine leishmaniasis," Advances in Parasitology, vol. 57, pp. 1-88, 2004.

[78] L. Ferrer, "Clinical aspects of canine leishmaniosis: an update," in Proceedings of the International Canine Leishmaniasis Forum, pp. 6-10, Hoechst Roussel Vet, Barcelona, Spain, 1999.

[79] C. Maia and L. Campino, "Methods for diagnosis of canine leishmaniasis and immune response to infection," Veterinary Parasitology, vol. 158, no. 4, pp. 274-287, 2008.

[80] P. F. Quaresma, S. M. F. Murta, E. de Castro Ferreira, A. C. V. M. da Rocha-Lima, A. A. P. Xavier, and C. M. F. Gontijo, "Molecular diagnosis of canine visceral leishmaniasis: identification of Leishmania species by PCR-RFLP and quantification of parasite DNA by real-time PCR," Acta Tropica, vol. 111, no. 3, pp. 289294, 2009.

[81] L. Manna, S. Reale, E. Viola et al., "Leishmania DNA load and cytokine expression levels in asymptomatic naturally infected dogs," Veterinary Parasitology, vol. 142, no. 3-4, pp. 271-280, 2006.

[82] L. Manna, S. Reale, E. Picillo, F. Vitale, and A. E. Gravino, "Urine sampling for real-time polymerase chain reactionbased diagnosis of canine leishmaniasis," Journal of Veterinary Diagnostic Investigation, vol. 20, no. 1, pp. 64-67, 2008.

[83] L. Solano-Gallego, A. Rodriguez-Cortes, M. Trotta et al., "Detection of Leishmania infantum DNA by fret-based realtime PCR in urine from dogs with natural clinical leishmaniosis," Veterinary Parasitology, vol. 147, no. 3-4, pp. 315-319, 2007.

[84] A. Franceschi, V. Merildi, G. Guidi, and F. Mancianti, "Occurrence of leishmania DNA in urines of dogs naturally infected with leishmaniasis," Veterinary Research Communications, vol. 31, no. 3, pp. 335-341, 2007. 

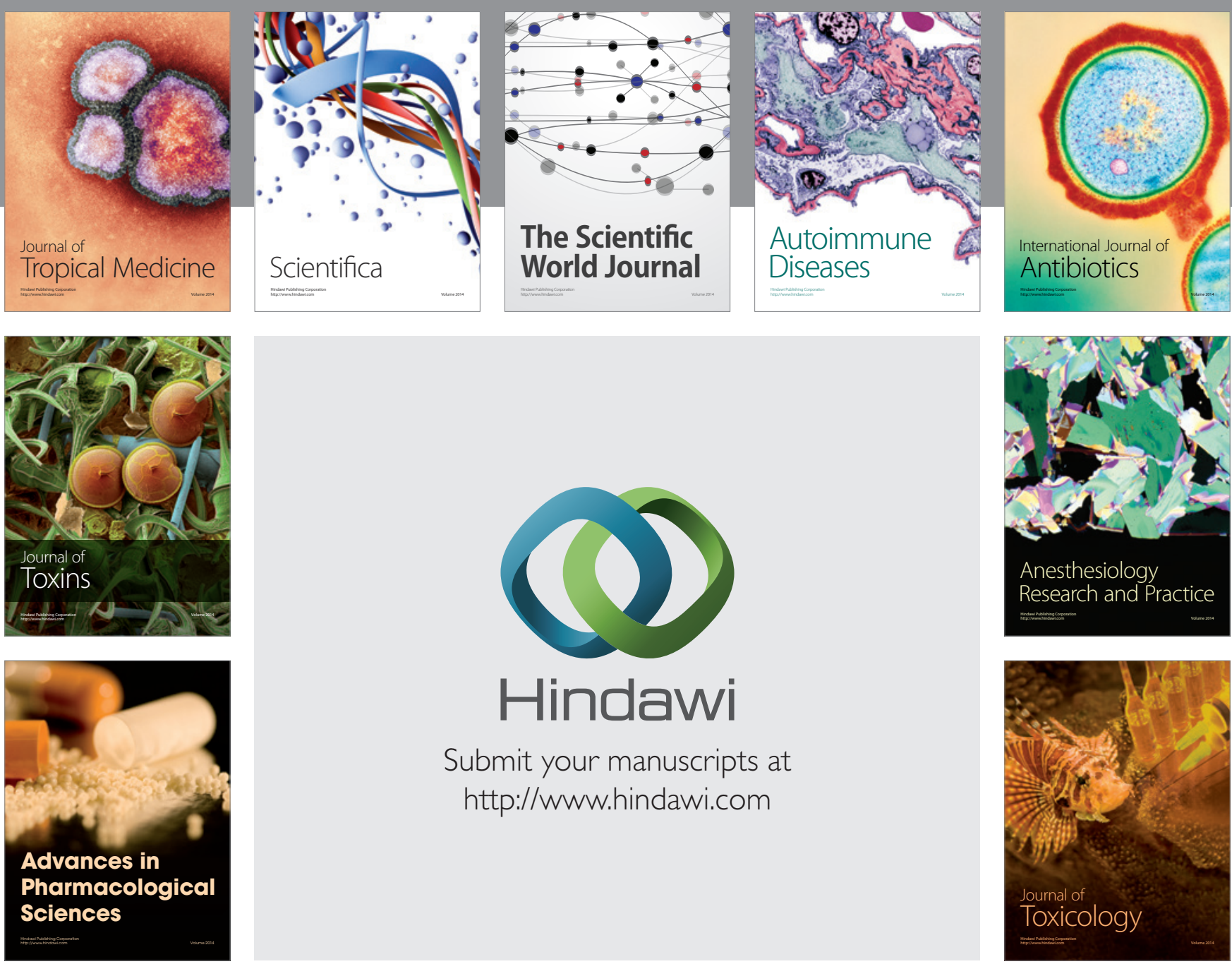

\section{Hindawi}

Submit your manuscripts at

http://www.hindawi.com
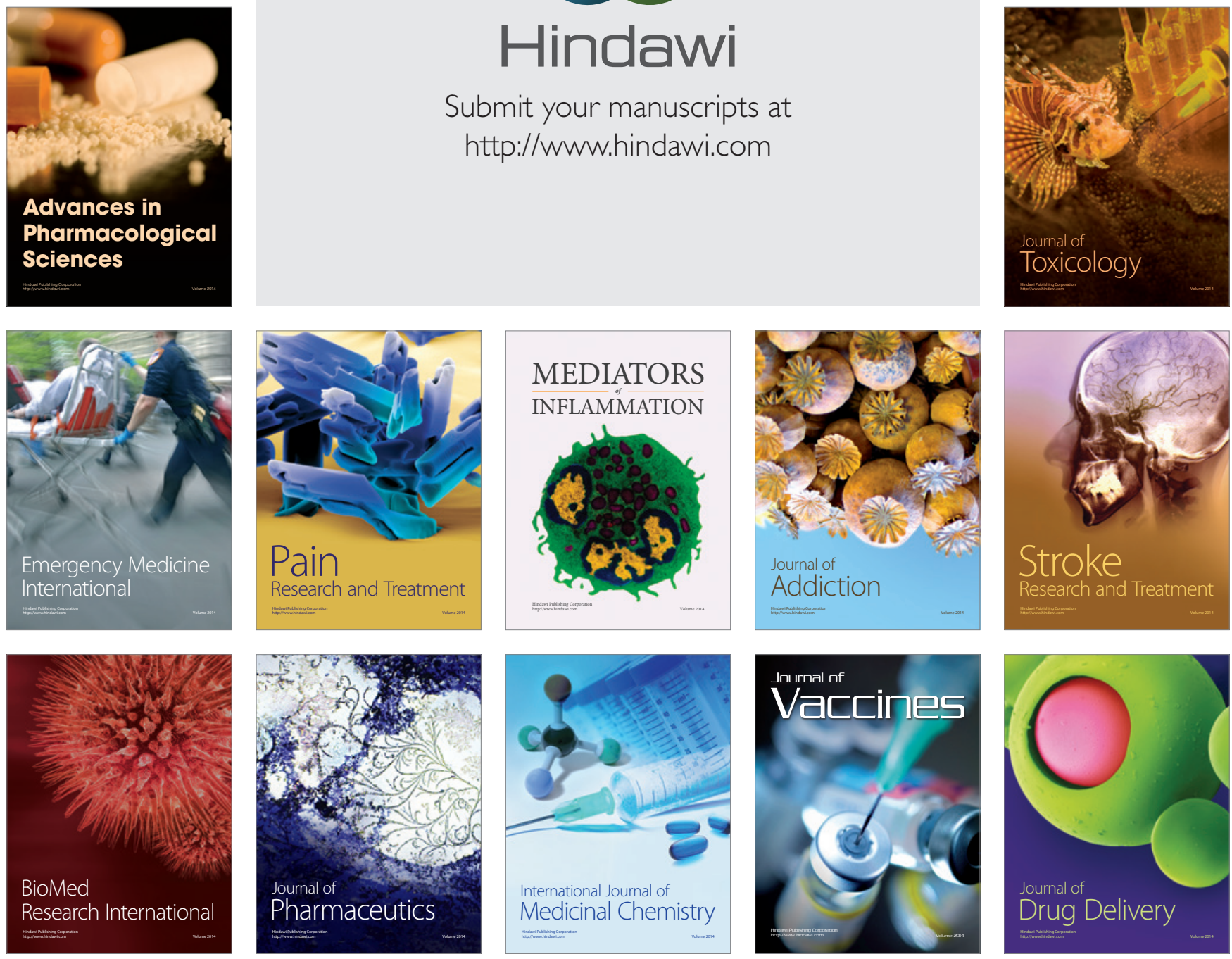